\begin{tabular}{ll|l}
\cline { 2 - 3 } & \multicolumn{3}{l}{ Intervent Neurol 2015;4:104-112 } \\
\cline { 2 - 3 } & $\begin{array}{l}\text { DOI: 10.1159/000442717 } 2016 \text { S. Karger AG, Basel } \\
\text { Published online: January 27, } 2016\end{array}$ & $\begin{array}{l}\text { (c) } \\
\text { 1664-9737/16/0044-0104\$39.50/0 } \\
\text { www.karger.com/ine }\end{array}$ \\
\hline
\end{tabular}

\title{
Whole-Brain Computed Tomographic Perfusion Imaging in Acute Cerebral Venous Sinus Thrombosis
}

\author{
Maxim Mokin a, e Chelsey C. Ciambella ${ }^{a, e} \quad$ Muhammad W. Masud ${ }^{b}$ \\ Elad I. Levy ${ }^{a, c}$, d, e Kenneth V. Snyder ${ }^{a-e}$ Adnan H. Siddiqui ${ }^{a, c, d-f}$
}

Departments of a Neurosurgery, ${ }^{b}$ Neurology and ${ }^{\mathrm{C}}$ Radiology, School of Medicine and Biomedical Sciences, and ${ }^{\mathrm{d}}$ Toshiba Stroke and Vascular Research Center, University at Buffalo, State University of New York, ${ }^{e}$ Department of Neurosurgery, Gates Vascular Institute/Kaleida Health, and fJacobs Institute, Buffalo, N.Y., USA

\section{Key Words}

Computed tomographic perfusion imaging · Venous sinus thrombosis

\begin{abstract}
Background: Acute cerebral venous sinus thrombosis (VST) can be difficult to diagnose because of its diverse clinical presentation. The utility of perfusion imaging for diagnosing VST is not well understood. Summary: We retrospectively reviewed cases of acute VST in patients who underwent whole-brain (320-detector-row) computed tomographic (CT) perfusion imaging in combination with craniocervical $C T$ venography. Perfusion maps that were analyzed included cerebral blood volume (CBV), cerebral blood flow (CBF), mean transit time, and time to peak. Among the 10 patients with acute VST included in this study, 9 had perfusion abnormalities. All perfusion abnormalities were localized in areas adjacent to the occluded sinus and did not match typical anterior or posterior circulation arterial territories. Bilateral perfusion deficits were seen in 4 cases. In 2 cases, parenchymal hemorrhage was diagnosed on noncontrast CT imaging; in those cases, focal CBV and CBF were reduced. Key Messages: Whole-brain CT perfusion imaging with 320-detector-row scanners can further assist in establishing the diagnosis of VST by detecting perfusion abnormalities corresponding to venous and not arterial territories. CT perfusion could assist in the differentiation between focal reversible changes, such as those caused by vasogenic edema, and irreversible changes due to infarction.

(C) 2016 S. Karger AG, Basel
\end{abstract}




\section{Introduction}

Acute cerebral venous sinus thrombosis (VST) is a rare form of stroke, and establishing a correct diagnosis can be challenging due to its diverse and typically delayed clinical presentation $[1,2]$. VST is most common in young women and is associated with pregnancy/puerperium, use of hormonal contraceptives, hypercoagulable states, and malignancy $[3,4]$. Overall mortality rates are low, in the range of $2-4 \%$, as a result of widespread treatment with systemic anticoagulation [5, 6]. Advanced age, focal neurologic deficits on admission, and comorbidities such as malignancy, autoimmune diseases, and substance abuse are associated with an increased risk of mortality from VST [5-7]. Computed tomographic (CT) venography and magnetic resonance (MR) venography are commonly used to visualize the cerebral venous system and confirm the diagnosis of VST, but the imaging value of these tests can be limited by anatomic variations of normal venous anatomy and imaging artifacts $[8,9]$.

Perfusion imaging allows the detection of ischemic brain tissue and is commonly used for the evaluation of patients with acute ischemic stroke [10]. The utility of perfusion imaging in diagnosing VST is not well understood. A 320-detector-row CT perfusion imaging scanner is capable of visualizing changes in the blood flow of the entire brain, including posterior circulation structures as well as cortical areas close to the vertex [11]. Here, we review CT perfusion findings from a cohort of patients with acute VST to determine perfusion map patterns specific to this disease.

\section{Methods}

After receiving approval from the University at Buffalo Health Sciences Institutional Review Board (project No. 578702-2), we retrospectively reviewed the medical records of patients with acute VST hospitalized between January 1, 2010 and October 1, 2013. At our institution, patients in whom VST is suspected undergo evaluation with noncontrast brain CT scanning followed by whole-brain (320-detector-row) CT perfusion imaging in combination with craniocervical CT venography, unless contraindications exist (in those cases, MR venography is performed). The diagnosis of VST is made on the basis of clinical presentation and confirmed by imaging tests, including noncontrast brain CT imaging and CT venography. The following data were collected for the present study: age, gender, description of clinical presentation, extent and location of VST, and CT perfusion findings.

\section{Imaging Protocol}

All patients underwent 5-mm noncontrast head CT imaging performed using an Aquilion ONE scanner (Toshiba Medical Systems, Nasu, Japan). CT perfusion was performed using a 320-detector-row CT system (Aquilion ONE). Contrast medium (50 ml, Optiray 350, Mallinckrodt, Mo., USA) was infused at a rate of $5 \mathrm{ml} / \mathrm{s}$ via automated antecubital venous injection. CT perfusion acquisition parameters were $80 \mathrm{kV}$ tube voltage, 200 $\mathrm{mA}$ tube current, and 0.35-second rotation. Perfusion maps [cerebral blood flow (CBF), cerebral blood volume (CBV), mean transit time (MTT), and time to peak (TTP)] were reconstructed utilizing Vitrea software (Version 6.4, Vital Images, Minnetonka, Minn., USA). Immediately afterward, craniocervical CT venography was performed using an infusion of contrast medium (80 ml, Omnipaque 350, GE Healthcare, Fairfield, Conn., USA) at a rate of $4 \mathrm{ml} / \mathrm{s}$. CT venography images were reconstructed at $0.5 \mathrm{~mm}$ thickness. The perceived perfusion abnormality was outlined manually and compared to the automatically generated contralateral region.

\section{Results}

We identified 10 patients with a diagnosis of acute VST who underwent CT perfusion as a part of the imaging evaluation. The mean age of these patients was 36 years (range 18-73 years), and $80 \%$ were women. Clinical presentation, imaging findings, treatment/proce- 


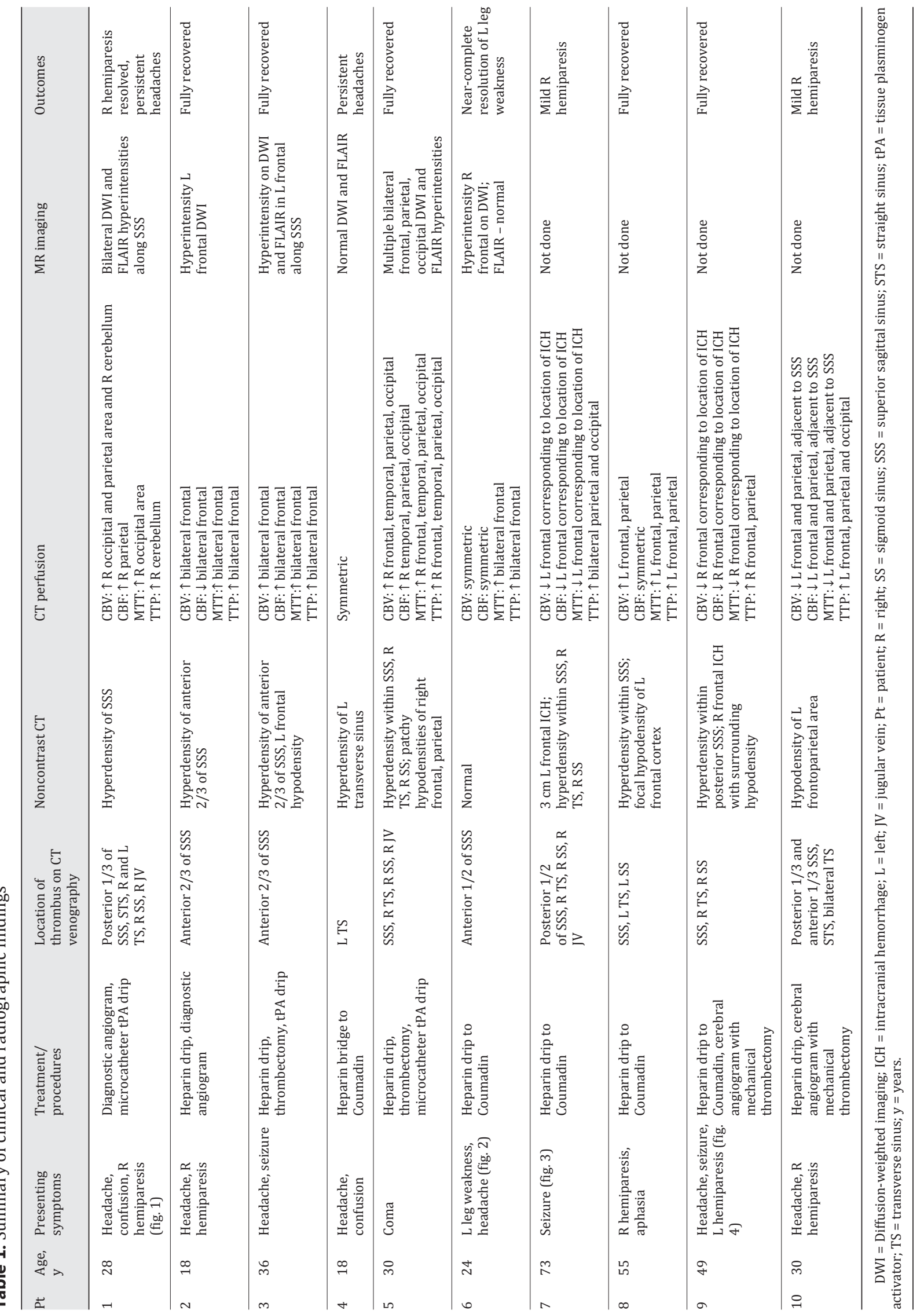


Mokin et al.: Whole-Brain Computed Tomographic Perfusion Imaging in Acute Cerebral Venous Sinus Thrombosis
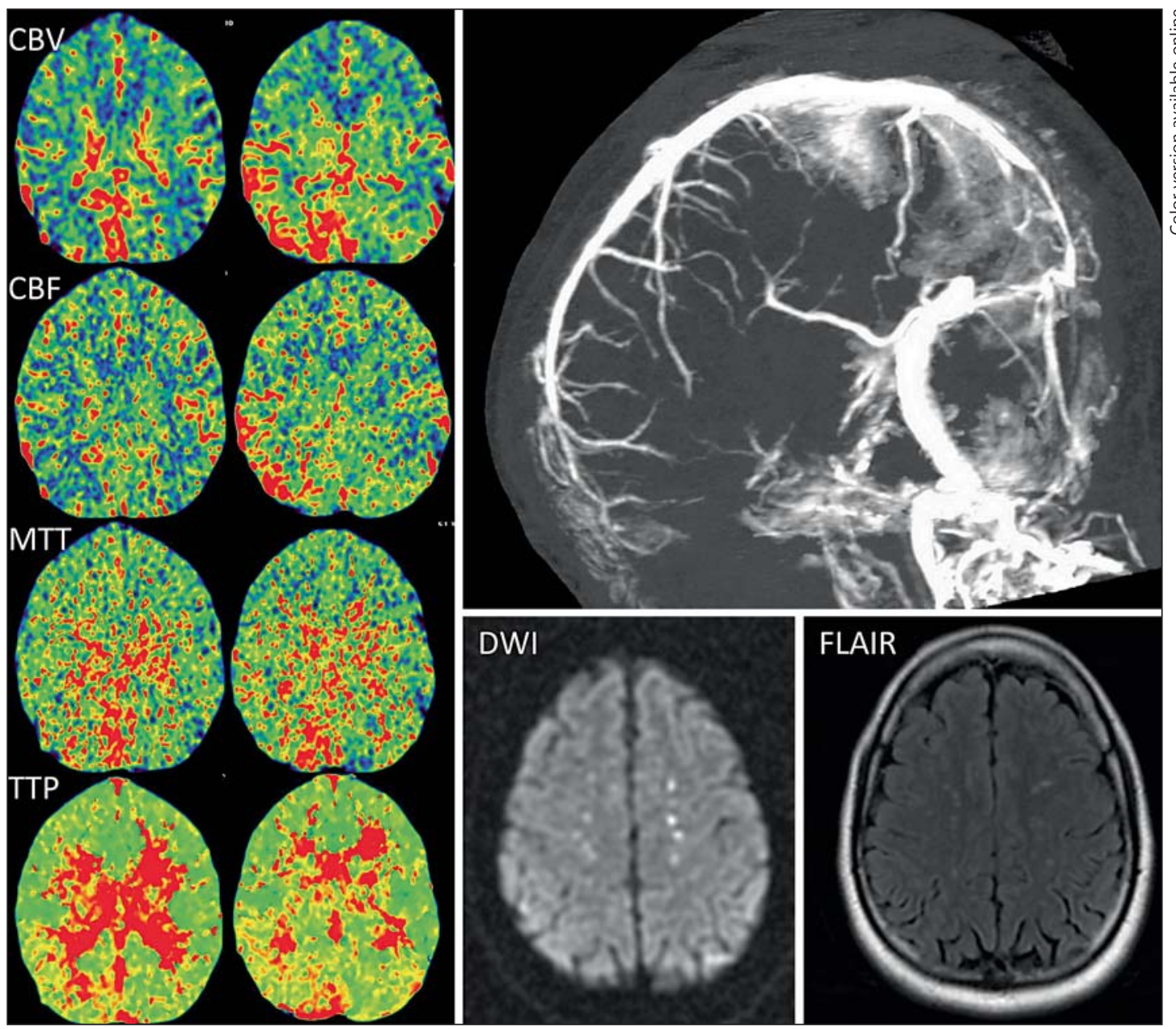

Fig. 1. CT perfusion maps (left) from case 1 demonstrate increased CBV and CBF in the right parieto-occipital area and the cerebellum adjacent to the superior sagittal and right transverse sinuses. There is also a delay in the MTT and TTP. CT venogram (top right) shows lack of flow within the posterior third of the superior sagittal sinus and bilateral transverse sinuses. The right sigmoid sinus and the jugular vein are also occlusive. MR imaging, diffusion-weighted imaging (DWI) and FLAIR sequences (bottom right) demonstrate bilateral hyperintensities along the occluded superior sagittal sinus.

dures, and outcomes are summarized in table 1 . The superior sagittal sinus was occlusive in 9 cases, the transverse sinus in 7 cases, the sigmoid sinus in 5 cases, the straight sinus in 2 cases, and the jugular vein in 3 cases.

Perfusion abnormalities were observed in all but 1 of the cases. Table 1 describes perfusion map findings for each individual case, and figures 1-4 provide examples of cases included in our series. CBV abnormality was observed in 8 cases, CBF abnormality in 7 cases, MTT abnormality in 9 cases, and TTP abnormality in 9 cases. All perfusion abnormalities were localized in the areas adjacent to the occluded sinuses and did not match typical anterior or posterior circulation arterial territories. Bilateral perfusion deficits were seen in 4 cases. In 2 cases, parenchymal hemorrhage was diagnosed on noncontrast CT; in those cases, focal CBV and CBF were reduced. 

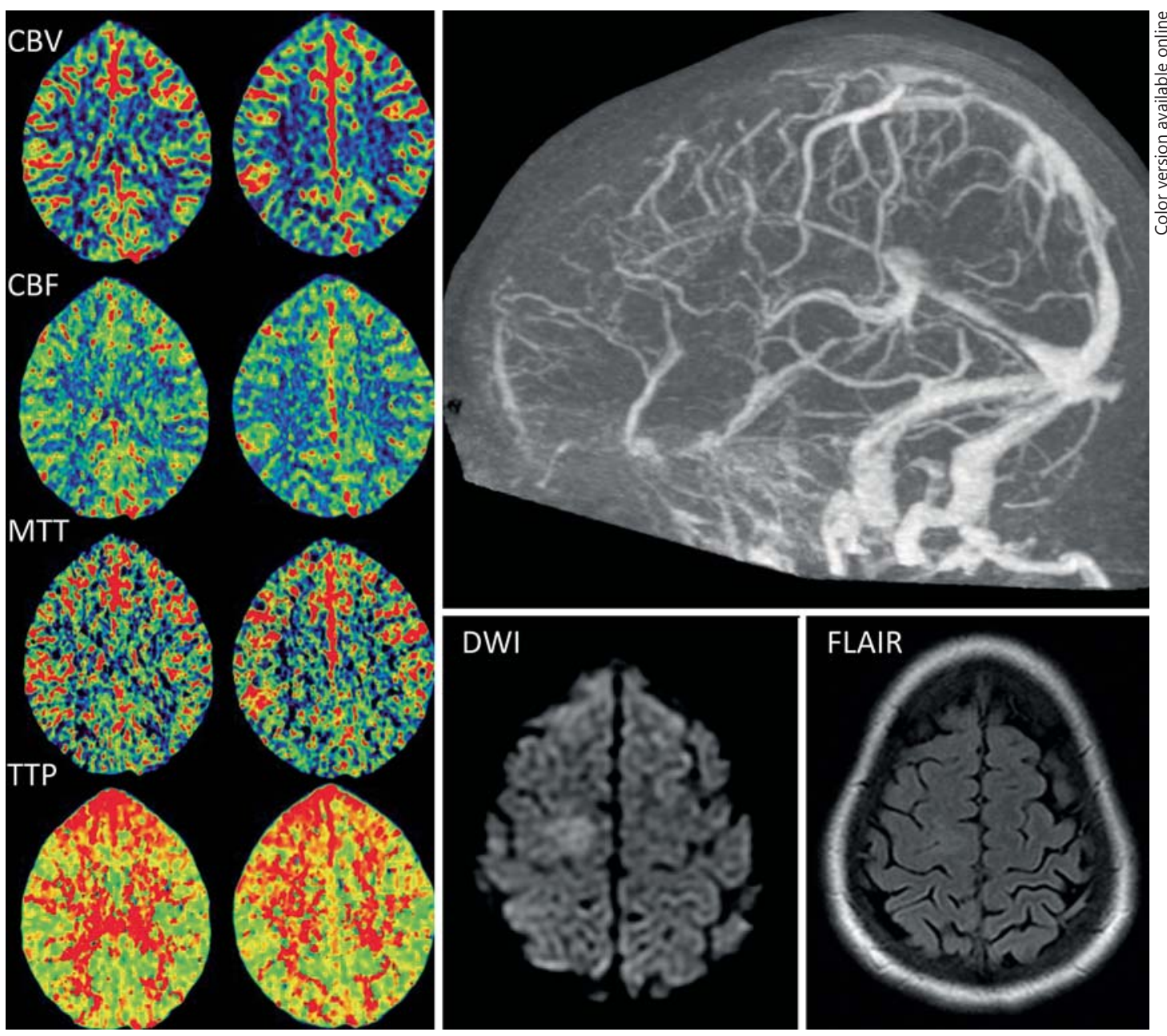

Fig. 2. CT perfusion maps (left) from case 6 demonstrate symmetric CBV and CBF. Increases in the MTT and TTP can be seen in the frontal areas bilaterally. Occlusion of the anterior half of the superior sagittal sinus is evident on the CT venogram (top right). Focal hyperintensity was seen on MR diffusion-weighted imaging (DWI) with unremarkable FLAIR sequence (bottom right).

\section{Discussion}

Abnormalities related to VST are frequently not detected through imaging of brain parenchyma. Previous studies using MR imaging for the diagnosis of VST have shown that focal changes caused by edema or infarction are found in only $25-50 \%$ of affected patients $[8,9$, $12]$. Both CT and MR venography are commonly used in clinical practice for the diagnosis of acute VST; unfortunately, both tests have drawbacks because of significant variability in normal venous anatomy variants and technical limitations $[9,12,13]$.

Perfusion imaging in acute VST is believed to identify both reversible and irreversible hemodynamic changes. Doege et al. [14] used MR perfusion imaging in 6 patients with acute VST and compared patterns of perfusion deficits with diffusion-weighted MR imaging changes. They concluded that an increase in MTT in the absence of changes in CBV and the MR imaging apparent diffusion coefficient was indicative of reversible parenchymal changes, analogous to the penumbra phenomenon in ischemic stroke. All patients had normal CBF values and all demonstrated excellent recovery, which suggests that the study likely excluded cases with 
Mokin et al.: Whole-Brain Computed Tomographic Perfusion Imaging in Acute Cerebral Venous Sinus Thrombosis
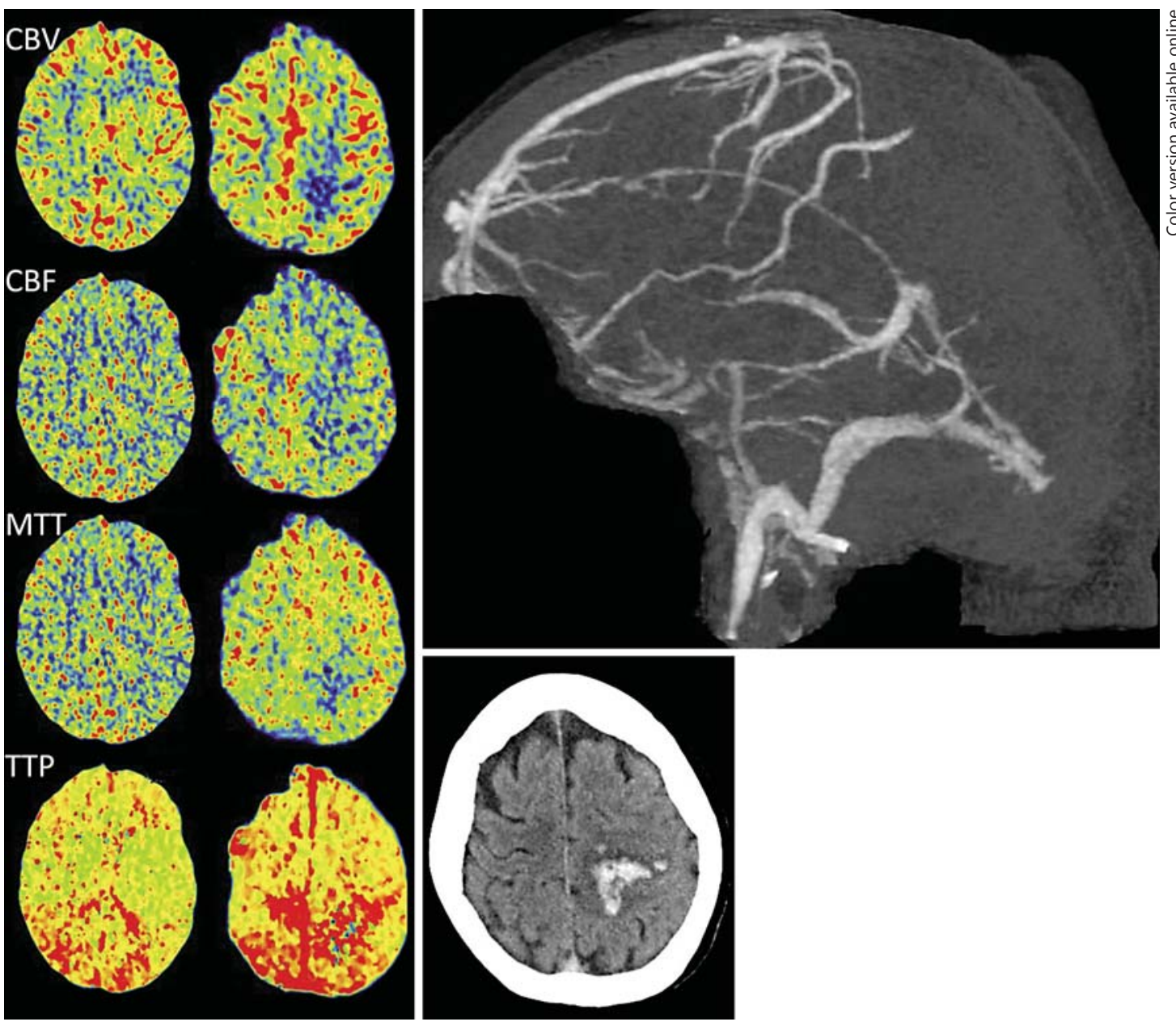

Fig. 3. CT perfusion maps (left) from case 7 show focally reduced CBV, CBF, and MTT in the left frontal area corresponding to the location of the hematoma originally seen on noncontrast brain CT imaging (lower right). The TTP is prolonged bilaterally. CT venogram shows occlusion of the superior sagittal, right transverse, and right sigmoid sinuses (upper right).

severe VST in which CBV changes could have occurred. Keller et al. [15] described a case of acute VST in which areas of vasogenic edema on MR imaging corresponded to increased regional CBV on MR perfusion.

Similarly, in one other case, elevated regional CBV was detected with the use of positron emission tomography [16]. Recanalization of the occluded superior sagittal sinus led to clinical improvement and correlated with either complete normalization or partial improvement of CBV values in previously affected territories.

Gupta et al. [17] used 4-slice CT perfusion in 20 patients with acute VST. Of those 20 patients, 16 had evidence of venous infarcts with hemorrhage and 4 had focal hypodensity on noncontrast CT or T1/fluid-attenuated inversion recovery (FLAIR) changes on MR imaging without hemorrhage. Perfusion abnormalities were detected in all 20 patients. CBV and CBF were reduced, and MTT was prolonged in both the core and periphery of lesions that were detected by noncontrast brain CT or MR imaging. Similarly, in our study, reduced $\mathrm{CBV}$ and $\mathrm{CBF}$ values were detected in 3 cases, of which 2 had parenchymal hematomas. Our results and the aforementioned studies suggest that $\mathrm{CBV}$ and $\mathrm{CBF}$ values might prove helpful 
Mokin et al.: Whole-Brain Computed Tomographic Perfusion Imaging in Acute Cerebral Venous Sinus Thrombosis
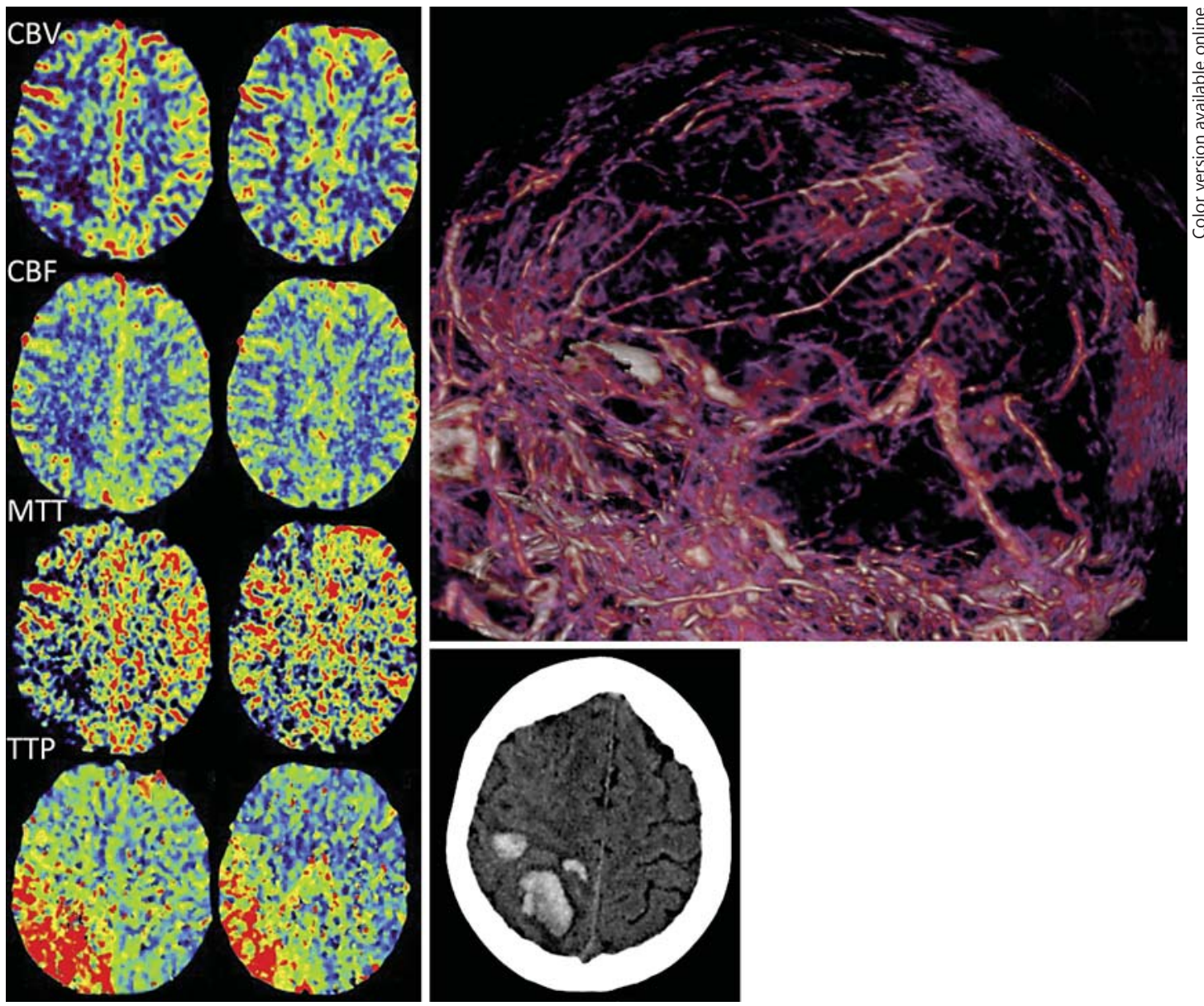

Fig. 4. CT perfusion maps (left) from case 9 display focally reduced CBV, CBF, and MTT in the right frontal area corresponding to the location of the hematoma that is shown on noncontrast brain CT imaging (lower right). The TTP is increased in the right frontal and parietal regions. Thrombosis of the superior sagittal, right transverse, and right sigmoid sinuses is seen on the CT venogram (upper right).

in the differentiation between focal reversible changes, such as those from vasogenic edema, and irreversible changes due to infarction. Data reported by Gupta et al. [17] also indicate a potential role for $\mathrm{CBV}$ and $\mathrm{CBF}$ values in the prediction of clinical outcomes for patients with VST.

Posterior fossa structures and those close to the vertex are typically not included in the field obtained with conventional CT or MR perfusion scanners, unless specifically requested by a clinician. Whole-brain CT perfusion imaging achieved by 320 -detector-row scanners can further assist in establishing the diagnosis of VST in clinically challenging cases by detecting perfusion abnormalities corresponding to venous and not arterial territories.

Anatomical variations of dural venous sinuses are frequently encountered in clinical practice, at times making it challenging to differentiate between a physiologically hypoplastic or absent sinus and incidental arachnoid granulations versus acute sinus occlusion from thrombosis [18-21]. Digital subtraction angiography can sometimes help establish the correct diagnosis by demonstrating dynamics of venous blood return, but this test is invasive and can also be inconclusive. Whole-brain CT perfusion detects regions with focal perfusion abnormality as a result of acute arterial occlusion (lack of inflow) or venous occlusion (lack 
Mokin et al.: Whole-Brain Computed Tomographic Perfusion Imaging in Acute

Cerebral Venous Sinus Thrombosis

of outflow). Therefore, correlation of regional venous sinus obstruction with the corresponding anatomic area of the perfusion abnormality will point towards an acute process, rather than an incidental anatomical variant.

Tsai et al. [22] analyzed outcomes in patients treated with endovascular pharmacological thrombolysis and mechanical thrombectomy (with and without the preceding administration of systemic anticoagulation) where the decision to escalate treatment was based on the combination of clinical symptoms and imaging evidence of cerebral venous congestion on CT and MR imaging. After comparing their outcomes to those of patients treated with systemic anticoagulation only, the authors proposed that indications for catheter-based thrombectomy should include both clinical (worsening or severe symptoms) and imaging (intracranial hemorrhage and edema) correlates of cerebral venous congestion.

Whether findings of regional flow abnormalities on CT perfusion should be included in the decision-making protocol when selecting a specific treatment modality for VST is to be determined. In our series of patients, CT perfusion was used as a tool to aid in establishing the correct diagnosis, whereas the decision for treatment was based on clinical response to systemic anticoagulation, based on current guidelines [8]. It would be interesting to know how treatment selected on the basis of both clinical and imaging-based criteria would affect patient outcomes.

The limitations of our study include its retrospective nature, lack of baseline MR imaging in patients 7-10, and descriptive rather than quantitative analysis of local CT perfusion map abnormalities. Also, post-treatment follow-up CT perfusion studies would have been of interest to see any resolution or persistence of original perfusion deficits but were not performed. Additional information gained with CT perfusion should be weighed against additional radiation dose, intravenous contrast material, and procedural costs.

\section{Conclusions}

Whole-brain CT perfusion imaging achieved by 320-detector-row scanners can further assist in establishing the diagnosis of VST by detecting perfusion abnormalities corresponding to venous and not arterial territories. CT perfusion could assist in the differentiation between focal reversible changes, such as those from vasogenic edema, and irreversible changes due to infarction.

\section{Acknowledgments}

The authors thank Paul H. Dressel BFA for the preparation of the illustrations and Debra J. Zimmer for editorial assistance. There was no funding/grant support in conjunction with this work.

\section{Disclosure Statement}

Ciambella and Masud: nothing to disclose. Levy: shareholder/ownership interests - Intratech Medical Ltd., Blockade Medical LLC, Medina Medical; national principal investigator - Covidien US SWIFT PRIME Trials; consultant - Pulsar, Medina Medical, Blockade Medical LLC; honoraria for training and lecturing Covidien; other financial support - Abbott for carotid training for physicians; Advisory Board - Stryker, NeXtGen Biologics. Mokin: educational grant - Toshiba. Siddiqui: research grants - the National Institutes of Health (co-investigator: NINDS 1R01NS064592-01A1, hemodynamic induction of pathologic remodeling leading to intracranial aneurysms), the National Institutes of Health (co-investigator: NIBIB 5 R01 EB002873-07, micro-radiographic image for neurovascular interventions), the National Institutes of Health (co-investigator: NIH/NINDS 1R01NS091075 virtual intervention of intracranial aneurysms); financial 
Mokin et al.: Whole-Brain Computed Tomographic Perfusion Imaging in Acute

Cerebral Venous Sinus Thrombosis

interests - Hotspur, Intratech Medical, StimSox, Valor Medical, Blockade Medical, Lazarus Effect, Pulsar Vascular, Medina Medical; consultant - Codman \& Shurtleff, Covidien Vascular Therapies, GuidePoint Global Consulting, Penumbra, Stryker, Pulsar Vascular, MicroVention, Lazarus Effect, Blockade Medical, Reverse Medical, W.L. Gore \& Associates; National Steering Committees - Penumbra-3D Separator Trial, CovidienSWIFT PRIME Trial, MicroVention-FRED Trial; speakers' bureau - Codman \& Shurtleff, Inc.; advisory board - Codman \& Shurtleff, Covidien Neurovascular, ICAVL, Medina Medical; honoraria - Penumbra, Toshiba Medical Systems. Snyder: research and stockholder - Boston Scientific; research and financial interest Cordis; research and financial interest - EndoTex; research and consultant support - Medtronic; research and consultant support - Abbott Vascular; research and consultant support - ev3; research and consultant support - Toshiba; research and consultant support and financial interest - Micrus; research and consultant support - Zimmer; financial interest and stockholder - Access Closure Inc.; stockholder - Niagara Gore Medical; research and financial interest - EPI; financial interest - Primus; research - Guidant; research Kerberos.

\section{References}

1 Gulati D, Strbian D, Sundararajan S: Cerebral venous thrombosis: diagnosis and management. Stroke 2014; 45:e16-e18.

2 Bushnell C, Saposnik G: Evaluation and management of cerebral venous thrombosis. Continuum (Minneap Minn) 2014;20:335-351.

3 Khealani BA, Wasay M, Saadah M, et al: Cerebral venous thrombosis: a descriptive multicenter study of patients in Pakistan and Middle East. Stroke 2008;39:2707-2711.

4 Wasay M, Bakshi R, Bobustuc G, et al: Cerebral venous thrombosis: analysis of a multicenter cohort from the United States. J Stroke Cerebrovasc Dis 2008;17:49-54.

5 Canhao P, Ferro JM, Lindgren AG, et al: Causes and predictors of death in cerebral venous thrombosis. Stroke 2005;36:1720-1725.

6 Nasr DM, Brinjikji W, Cloft HJ, et al: Mortality in cerebral venous thrombosis: results from the national inpatient sample database. Cerebrovasc Dis 2013;35:40-44.

7 Girot M, Ferro JM, Canhao P, et al: Predictors of outcome in patients with cerebral venous thrombosis and intracerebral hemorrhage. Stroke 2007;38:337-342.

8 Saposnik G, Barinagarrementeria F, Brown RD Jr, et al; the American Heart Association Stroke Council and the Council on Epidemiology Prevention: Diagnosis and management of cerebral venous thrombosis: a statement for healthcare professionals from the American Heart Association/American Stroke Association. Stroke 2011; 42:1158-1192.

9 Leach JL, Fortuna RB, Jones BV, et al: Imaging of cerebral venous thrombosis: current techniques, spectrum of findings, and diagnostic pitfalls. Radiographics 2006;26(suppl 1):S19-S43.

10 Latchaw RE, Alberts MJ, Lev MH, et al: Recommendations for imaging of acute ischemic stroke: a scientific statement from the American Heart Association. Stroke 2009;40:3646-3678.

11 Snyder KV, Mokin M, Bates VE: Neurologic applications of whole-brain volumetric multidetector computed tomography. Neurol Clin 2014;32:237-251.

12 Sajjad Z: MRI and MRV in cerebral venous thrombosis. J Pak Med Assoc 2006;56:523-526.

13 Janjua N: Cerebral angiography and venography for evaluation of cerebral venous thrombosis. J Pak Med Assoc 2006; 56:527-530.

14 Doege CA, Tavakolian R, Kerskens CM, et al: Perfusion and diffusion magnetic resonance imaging in human cerebral venous thrombosis. J Neurol 2001;248:564-571.

15 Keller E, Flacke S, Urbach H, et al: Diffusion- and perfusion-weighted magnetic resonance imaging in deep cerebral venous thrombosis. Stroke 1999;30:1144-1146.

16 Kawai N, Shindou A, Masada T, et al: Hemodynamic and metabolic changes in a patient with cerebral venous sinus thrombosis: evaluation using 0-15 positron emission tomography. Clin Nucl Med 2005;30:391-394.

17 Gupta RK, Bapuraj JR, Khandelwal N, et al: Prognostic indices for cerebral venous thrombosis on CT perfusion: a prospective study. Eur J Radiol 2014;83:185-190.

18 Leach JL, Meyer K, Jones BV, et al: Large arachnoid granulations involving the dorsal superior sagittal sinus: findings on MR imaging and MR venography. AJNR Am J Neuroradiol 2008;29:1335-1339.

19 San Millan Ruiz D, Fasel JH, Gailloud P: Unilateral hypoplasia of the rostral end of the superior sagittal sinus. AJNR Am J Neuroradiol 2012;33:286-291.

20 Silva PS, Vilarinho A, Carvalho B, et al: Anatomical variations of the vein of Labbe: an angiographic study. Surg Radiol Anat 2014;36:769-773.

21 Surendrababu NR, Subathira, Livingstone RS: Variations in the cerebral venous anatomy and pitfalls in the diagnosis of cerebral venous sinus thrombosis: low field MR experience. Indian J Med Sci 2006;60:135-142.

22 Tsai FY, Kostanian V, Rivera M, et al: Cerebral venous congestion as indication for thrombolytic treatment. Cardiovasc Intervent Radiol 2007;30:675-687. 